Article

\title{
Genome-Wide Association Study of Diabetogenic Adipose Morphology in the GENetics of Adipocyte Lipolysis (GENiAL) Cohort
}

\author{
Veroniqa Lundbäck ${ }^{1}$, Agné Kulyté ${ }^{1}$, Peter Arner ${ }^{1}$, Rona J. Strawbridge ${ }^{2,3,4,+}$ (i) and \\ Ingrid Dahlman $1, *,+(\mathbb{D}$ \\ 1 Lipid laboratory, Endocrinology Unit, Department of Medicine Huddinge, Karolinska Institutet, \\ 171-77 Stockholm, Sweden; veroniqa.lundback@ki.se (V.L.); agne.kulyte@ki.se (A.K.); peter.arner@ki.se (P.A.) \\ 2 Institute of Health and Wellbeing, University of Glasgow, College of Medicine, \\ Veterinarian and Life Sciences, Glasgow G12-8RZ, UK; Rona.Strawbridge@glasgow.ac.uk \\ 3 Department of Medicine Solna, Karolinska Institutet, 171-77 Stockholm, Sweden \\ 4 Health Data Research University of Glasgow, College of Medicine, Veterinarian and Life Sciences, \\ Glasgow G12-8RZ, UK \\ * Correspondence: ingrid.dahlman@ki.com \\ + These authors have contributed equally to this work.
}

Received: 23 March 2020; Accepted: 23 April 2020; Published: 27 April 2020

\begin{abstract}
An increased adipocyte size relative to the size of fat depots, also denoted hypertrophic adipose morphology, is a strong risk factor for the future development of insulin resistance and type 2 diabetes. The regulation of adipose morphology is poorly understood. We set out to identify genetic loci associated with adipose morphology and functionally evaluate candidate genes for impact on adipocyte development. We performed a genome-wide association study (GWAS) in the unique GENetics of Adipocyte Lipolysis (GENiAL) cohort comprising 948 participants who have undergone abdominal subcutaneous adipose biopsy with a determination of average adipose volume and morphology. The GWAS identified 31 genetic loci displaying suggestive association with adipose morphology. Functional evaluation of candidate genes by small interfering RNAs (siRNA)-mediated knockdown in adipose-derived precursor cells identified six genes controlling adipocyte renewal and differentiation, and thus of potential importance for adipose hypertrophy. In conclusion, genetic and functional studies implicate a regulatory role for ATL2, ARHGEF10, CYP1B1, TMEM200A, C17orf51, and $L 3 M B T L 3$ in adipose morphology by their impact on adipogenesis.
\end{abstract}

Keywords: adipose morphology; adipogenesis; genome-wide association study (GWAS); genetic loci; GENetics of Adipocyte Lipolysis (GENiAL) cohort

\section{Introduction}

Excess adipose tissue plays a central role in the risk of developing insulin resistance (IR) and, thereby, the development of type 2 diabetes (T2D). However, it is not only the size but also the morphology of subcutaneous adipose tissue (SAT), which influences adipose function [1,2]. The number of fat cells differs substantially between individuals, even among those with the same total body fat, giving rise to hypertrophic, i.e., fewer but larger fat cells, or hyperplastic, i.e., more but smaller fat cells, adipose morphology [3,4]. Hypertrophic SAT is the pernicious morphology and has been associated with IR and T2D [5,6]. Hypertrophic SAT has been linked to local IR and increased release of pro-inflammatory mediators and free fatty acids within SAT $[5,6]$, which through systemic effects, contribute to systemic IR. 
The factors governing SAT hyperplastic versus hypertrophic expansion is poorly understood. Human SAT contains a pool of progenitor cells that are continuously recruited to the adipose lineage to mature adipocytes [3]. Hypertrophic adipose morphology has been associated with reduced numbers of new adipocytes [4,6] and altered extracellular matrix function [7]. Adipocytes are turned over slowly [4], and adipose morphology is, thus, a relatively stable phenotype over time. Importantly, the observation that bariatric surgery induces a hyperplastic adipose morphology, which is associated with beneficial systemic metabolic parameters in comparison to body mass index (BMI)-matched controls, gives support to the notion that treatments improving adipose morphology have potential as therapeutic targets for IR and T2D [8].

First degree relatives of patients with T2D have been characterized as having hypertrophic adipose tissue consistent with a genetic impact on adipose morphology and link to IR/T2D [9]. Recently, large genome-wide association studies (GWAS) have identified hundreds of genetic risk loci for IR and T2D, and have reported that subjects carrying certain risk alleles exhibit reduced SAT indicating that inability of proper SAT expansion and body fat distribution are important contributing factors for the risk of developing metabolic disease [10]. There is evidence that body fat distribution and adipose morphology are related [7]. However, to the best of our knowledge, up to now, no GWAS has directly addressed the genetic regulation of adipose morphology, and its role in development of IR/T2D.

In the present study, we conducted a GWAS for adipose morphology to identify genetic loci associated with, and highlight potential variants influencing adipose morphology. Adipose morphology was assessed using average adipocyte size in the abdominal SAT region adjusted for the size of the fat depot; a positive value is indicative of larger than expected adipocytes in relation to body fat mass (hypertrophy), and a negative value indicates hyperplasia [11]. Candidate genes in adipose morphology-associated loci were taken forward for functional evaluation, with in vitro experiments in adipocytes being used to determine the impact on phenotypes central to the development of adipose hypertrophy, i.e., precursors cell proliferation and adipocyte differentiation.

\section{Materials and Methods}

\subsection{Participants}

The GENiAL cohort has been described previously [12]. In the present study, we included all 948 participants for which SAT adipocyte size had been measured to determine adipose morphology (Supplementary Figure S1). Descriptives of the study subjects are presented in Table 1. Fifty-seven percent were obese (defined as BMI $\geq 30 \mathrm{~kg} / \mathrm{m}^{2}$ ). Total body fat mass was indirectly calculated using a formula based on age, sex, and BMI [13]. A venous blood sample was obtained for the extraction of DNA and clinical chemistry analyses by the hospital's accredited routine clinical chemistry laboratory. All participants lived in Stockholm county, Sweden. One hundred and ninety-four participants had type 2 diabetes, hypertension, or dyslipidemia alone or in different combinations. None were treated with insulin, glitazones, or glucagon-like-peptide analogs. Data on clinical and adipose variables have been published previously $[14,15]$. The study was approved by the local committee on ethics at the Huddinge University Hospital (D. no. 167/02, 2002-06-03) and explained in detail to each participant. Informed consent was obtained from all participants; this was in written form since 1996.

SAT gene expression was studied in 114 women, which is a subset of the above cohort. This cohort has been described previously [16] and contained the same type of adipose data as presented herein, with the exception that adipose morphology was calculated by relating average adipose volume to total body fat. 
Table 1. Characteristics of participants in the GENetics of Adipocyte Lipolysis (GENiAL) cohort used for the analysis of adipose morphology.

\begin{tabular}{ccccccc}
\hline & & Men & & & Women & \\
& Non Obese & Obese & $\boldsymbol{P}$ & Non Obese & Obese & $\boldsymbol{P}$ \\
\hline lean/obese (n) & 155 & 91 & & 261 & 441 & \\
age (years) & $47(14)$ & $43(12)$ & 0.01 & $40(13)$ & $41(10)$ & 0.38 \\
height (cm) & $179(6)$ & $181(7)$ & 0.014 & $167(6)$ & $166(7)$ & 0.0044 \\
body weight (kg) & $81(9)$ & $125(18)$ & $<0.0001$ & $67(9)$ & $108(17)$ & $<0.0001$ \\
BMI (kg/m $\left.{ }^{2}\right)$ & $25(2)$ & $38(5)$ & $<0.0001$ & $24(3)$ & $39(5)$ & $<0.0001$ \\
waist (cm) & $93(8)$ & $126(13)$ & $<0.0001$ & $84(10)$ & $119(13)$ & $<0.0001$ \\
WHR & $0.95(0.06)$ & $1.05(0.05)$ & $<0.0001$ & $0.86(0.07)$ & $0.96(0.07)$ & $<0.0001$ \\
systolic blood pressure (mm Hg) & $129(15)$ & $140(18)$ & $<0.0001$ & $120(16)$ & $130(16)$ & $<0.0001$ \\
diastolic blood pressure (mm Hg) & $79(10)$ & $86(12)$ & $<0.0001$ & $74(10)$ & $79(10)$ & $<0.0001$ \\
plasma total cholesterol (mmol/L) & $5.2(1.3)$ & $5.3(1.4)$ & 0.4 & $4.9(1.1)$ & $5.0(1.0)$ & 0.13 \\
plasma HDL cholesterol (mmol/L) & $1.3(0.4)$ & $1.0(0.2)$ & $<0.0001$ & $1.6(0.4)$ & $1.2(0.3)$ & $<0.0001$ \\
plasma triacylglycerides (mmol/l) & $1.72(2.1)$ & $2.36(2.52)$ & 0.044 & $1.07(0.64)$ & $1.53(0.87)$ & $<0.0001$ \\
plasma non-esterified fatty acids & $0.49(0.17)$ & $0.61(0.21)$ & $<0.0001$ & $0.62(0.21)$ & $0.72(0.24)$ & $<0.0001$ \\
(mmol/L) & $60.4(27.7)$ & $78.5(30.2)$ & $<0.0001$ & $81.4(43.0)$ & $116.8(54.3)$ & $<0.0001$ \\
plasma glycerol (mmol/l) & $5.5(1.2)$ & $6.4(2.3)$ & 0.0012 & $5.0(0.7)$ & $5.6(1.4)$ & $<0.0001$ \\
fasting plasma glucose (mmol/L) & $7.9(5.3)$ & $20.8(11.8)$ & $<0.0001$ & $6.2(3.3)$ & $14.8(8.0)$ & $<0.0001$ \\
fasting serum insulin (mU/l) & $2.0(2.0)$ & $6.1(4.3)$ & $<0.0001$ & $1.4(0.9)$ & $3.8(2.9)$ & $<0.0001$ \\
HOMA-IR & $503(142$ & $826(192)$ & $<0.0001$ & $474(179)$ & $862(180)$ & $<0.0001$ \\
Cell volumn (pl) & $0.8(0.7)$ & $3.8(2.7)$ & $<0.0001$ & $1.0(0.9)$ & $3.4(2.9)$ & $<0.0001$ \\
Spontaneous lipolys (mmol & “6(128)" & $13(171$ & 0.36 & “-13(149) & $10(168)$ & 0.054 \\
glycerol/2hrs/ESAT) & & &
\end{tabular}

Where: obese is defined as BMI $>30 \mathrm{~kg} / \mathrm{m}^{2}$; Spontaneous lipolysis rate was calculated as glycerol release divided by the lipid weight of the incubated fat cells; adipose morphology is difference between the measured and expected mean adipocyte volume at the abdominal subcutaneous adipose tissue depot. continuous variables are presented as mean (SD), groups were compared with Student's $t$-test (unpaired). BMI, body mass index, WHR, waist hip ratio, HDL, high-density lipoprotein, HOMA-IR, homeostatic model assessment of insulin resistance, ESAT, estimated abdominal subcutaneous fat mass.

\subsection{Adipose Tissue Biopsy and Measurement of Adipose Morphology}

GENiAL participants were examined in the morning after an overnight fast. Following a clinical examination, an abdominal SAT biopsy was obtained by a needle aspiration biopsy lateral to the umbilicus as described [17]. SAT samples were rapidly rinsed in sodium chloride $(9 \mathrm{mg} / \mathrm{mL})$ before the removal of visual blood vessels and cell debris and subsequently subjected to collagenase treatment to obtain isolated adipocytes as described [18]. The mean weight and volume of adipocytes and their spontaneous lipolysis were determined as described $[19,20]$. A curve fit of the relationship between mean adipocyte volume and estimated abdominal subcutaneous fat mass (ESAT) was performed exactly as described [11]. This non-linear relationship for the GENiAL cohort is published in Andersson D et al. The difference between the measured and expected mean adipocyte volume at the corresponding fat mass determines adipose morphology [11]. If the measured adipocyte volume is larger than expected, SAT hypertrophy prevails, whereas the opposite is valid for hyperplasia. Thus, this measure of adipose morphology is independent of fat mass.

\subsection{Genetic Analysis}

Genotyping and quality control (QC) have been described [12]. After quality control, imputation was performed using the haplotype reference consortium panel and, when variants were not available, using the $1000 \mathrm{G}$ phase3 reference panel [21]. Post-imputation quality control excluded single nucleotide polymorphisms (SNPs) with minor allele count $<3$ and imputation quality information INFO $<0.4$ as well as related individuals (one of each pair of $1^{\text {st }}$ or $2^{\text {nd }}$-degree relatives). After quality control, 894 samples and 9,714,326 SNPs were available for phenotypic analysis. A GWAS was conducted in PLINK 2.0 [22] using linear regression, assuming an additive genetic model, and adjusting for population structure (PCs1-3), age, and sex. Genome-wide significance was set at $p<5 \times 10^{-8}$, 
and suggestive significance was set at $p<1 \times 10^{-5}$. Only SNPs with minor allele frequency (MAF) $>1 \%$ were included in the results. Results were visualized using FUMA [23]. Data for one SNP included in the herein described GWAS is included in a separate manuscript by Carl Herdenberg E et al., where leucine-rich repeats and immunoglobulin-like domains 1 (LRIG) proteins regulate lipid metabolism via bone morphogenic (BMP) signaling and affect the risk of type 2 diabetes (submitted). This SNP displays a nominal association only with adipose morphology and is, therefore, not included in the presentation of GWAS results in the present study.

\subsection{Data Mining}

SNPs reaching GWAS or suggestive significance were examined for genotype-specific gene expression in adipose tissue (expression quantitative locus; eQTL) using GTEx database on January 15, 2020 [24].

\subsection{Adipocyte Cell Culture and Transfection With Small Interfering RNA}

Isolation, growth, and differentiation of SAT-derived human mesenchymal stem cells (hMSCs) have been described previously [25]. Marker gene expression (Cap analysis gene expression (CAGE) and gene expression data) and lipid accumulation images for these cells have been published previously [26].

hMSCs were transfected using a Neon electroporator (Invitrogen, Carlsbad, CA, US) according to the manufacturer's protocol. Briefly, 1 million hMSCs at day -4 before initiation of differentiation were mixed with $40 \mathrm{nM}$ ON-TARGETplus SMARTpool small interfering RNAs (siRNAs) targeting genes of interest or non-targeting siRNA pool (Dharmacon, Lafayette, CO, US) and electroporated using a $100 \mu \mathrm{L}$ NEON electroporation tip. Electroporation conditions were 1150 Volts, 30 ms width, 2 pulses. Electroporation was repeated until the required number of cells were collected for the experimental set-up. Following electroporation, the cells were plated in an antibiotic-free medium at the density of 55,000 cells/well in 24-well plates or 5500 cells/well in 96-well plate and cultured up to day 9 of differentiation as specified below.

\subsection{Measurement of Lipid Accumulation and Lipolysis}

Lipid accumulation was quantified at differentiation day 9. hMSCs differentiated in vitro were washed with PBS and fixed with $4 \%$ paraformaldehyde solution for $10 \mathrm{~min}$ at room temperature. Following fixation, neutral lipids were stained with Bodipy 493/503 (at $0.2 \mu \mathrm{g} / \mathrm{mL}$; Molecular Probes, Thermo Fisher Scientific, Waltham, MA, US), and nuclei (DNA) were stained with Hoechst 33342 (at $2 \mu \mathrm{g} / \mathrm{mL}$; Molecular Probes) for $20 \mathrm{~min}$ at room temperature. Accumulation of neutral lipids and cell numbers were quantified in CellInsight ${ }^{\mathrm{TM}}$ CX5 High Content Screening (HCS) Platform (Thermo Fischer Scientific, Waltham, MA, US) with integrated "Spot detection" protocol. Total Bodipy fluorescence (lipid droplets) was normalized to the number of nuclei, representing the number of cells in each well.

The medium was collected at day 7 and 9 of differentiation of hMCSs for measuring glycerol release as an index of lipolysis, as described [27]. A standard curve ranging from 0 to $120 \mu \mathrm{M}$ was used to calculate the concentrations of the samples. Amounts of glycerol was normalized to the number of nuclei and in each well.

\subsection{Analysis of Cell Proliferation}

hMSCs were electroporated using $10 \mu \mathrm{l}$ NEON electroporation tip day -4 before initiation of differentiation. Electroporation conditions were 1150 Volts, $30 \mathrm{~ms}$ width, 2 pulses. Cells were incubated with media containing $5 \mu \mathrm{M}$ 5-ethynyl-2'-deoxyuridine (EdU) at day -3 for $24 \mathrm{~h}$, followed by an assessment of EdU-positive cells using the Click-iT ${ }^{\circledR}$ Plus EdU Alexa Fluor555 (C10352, Invitrogen, Carlsbad, CA). 


\subsection{Isolation of RNA and Analysis of Gene Expression}

Following electroporation of siRNA, hMSCs were collected for isolation of RNA at the day differentiation was induced (day 0), days 1, and 7 post-induction of differentiation. Extraction of total RNA, measurement of concentration and purity as well as reverse transcription were performed as described [27]. Quantitative RT-PCR of coding genes was performed using commercial TaqMan probes (Applied Biosystems, Foster City, US). Gene expression was normalized to the internal reference gene 18s. Relative expression was calculated using the $2(-\Delta \Delta$ threshold cycle) method [28].

\subsection{Statistical Analysis of Clinical and In Vitro Data}

Clinical and in vitro results are presented as mean \pm standard deviation (SD). Glycerol and triglycerides values were normalized by $\log 10$ transformation prior to analysis. Standard statistical tests were used, including $t$-test, single or multiple regression as indicated in figure/table legends using Stat View software (Abacus Concepts Inc, Berkley, CA) or JMP 14 (SAS, NC, USA).

\section{Results}

\subsection{Clinical Findings}

The demographic characteristics of the GENiAL cohort participants included in the GWAS of adipose morphology are presented in Table 1 . The cohort consisted predominantly of women, who were younger and had a higher frequency of obesity than the men. Obesity was associated with higher systemic levels of fasting glucose and insulin, and with a pernicious lipid profile in both sexes. Average abdominal subcutaneous adipocyte volume was larger in obese subjects, whereas adipose morphology, as expected, was unrelated to obesity status.

Adipose morphology was correlated with insulin resistance as estimated by fasting serum (fS)-Insulin (standardized beta 0.24, $p 2 \times 10^{-12}$ ), and HOMA-IR (standardized beta $0.16, p 1.5 \times 10^{-6}$ ) after adjusting for age and sex.

\subsection{GWAS for Adipose Morphology}

The results of the GWAS are presented in Supplementary Figure S1, Table 2, and Supplementary Table S1. A total of 66 SNPs in 31 loci showed suggestive association $\left(p<10^{-5}\right)$ with adipose morphology. Six genetic loci, each $<1 \mathrm{Mb}$, contained $\geq 3 \mathrm{SNPs}$ displaying suggestive association $\left(p<10^{-5}\right)$ with adipose morphology (Figure 1); subsequent analysis was focused on these genomic regions. The strongest association with adipose morphology was observed in a region on chromosome 3, where one SNP, rs2378515, displayed a borderline GWAS-significant association $\left(p 5.20 \times 10^{-8}\right)$. This SNP is situated in the TP63 gene (Figure 1), which was not expressed in mature adipocytes, nor in our hMSCs during in vitro differentiation (results not shown) and could, therefore, not be taken forward for functional evaluation. No other gene is encoded within this LD-block bounded by a recombination fraction $\sim 20 \%$ (Figure 1). On chromosome 2, we identified tag-SNP rs147711728 among multiple SNPs $(n=6)$ of suggestive significance in the region that harbors the ATL2, CYP1B1, and RMD2 genes. On chromosome 6, tag-SNP rs62431222 defines a locus, which includes eight SNPs reaching suggestive significance and harboring genes L3MBTL3, SAMD3, and TMEM200A. Chromosome 8, with tag-SNP rs11988258 and three other SNP with suggestive association, had genes CLN8 and ARHGEF10 in the locus. On chromosome 9, tag-SNP rs145072648 is in a locus containing the KLHL9 and the IFNA family of genes. The chromosome 17 locus includes three associated SNPs with tag-SNP rs201766885 and harbors FAM27L, MTRNR2L1, and C17orf51.

All 66 SNPs in Supplementary Table S1 were investigated in the GTEx eQTL resource, but failed to provide evidence for genotype-dependent gene expression in SAT. All SNPs resided in regions that do not encode protein and are, therefore, of unclear impact. 
Table 2. Tag SNPs associated with adipose morphology with $p<10^{-5}$.

\begin{tabular}{|c|c|c|c|c|c|c|c|c|}
\hline Chrom & POS & ID & $\begin{array}{l}\text { Effect } \\
\text { Allele }\end{array}$ & BETA & L95 & U95 & $P$ & A1_FREQ \\
\hline 1 & 3297459 & rs201839757 & I & 198 & 113 & 283 & 5.75E-06 & 0.0126 \\
\hline 1 & 48004858 & rs12032932 & $\mathrm{T}$ & 167 & 96 & 238 & $4.40 \mathrm{E}-06$ & 0.0110 \\
\hline 1 & 76545413 & rs10443175 & $\mathrm{T}$ & -40 & -57 & -22 & $8.15 \mathrm{E}-06$ & 0.2247 \\
\hline 1 & 157782519 & rs77346326 & $\mathrm{T}$ & 136 & 81 & 191 & $1.28 \mathrm{E}-06$ & 0.0203 \\
\hline 2 & 19739010 & rs3914966 & $\mathrm{T}$ & -34 & -48 & -19 & 4.64E-06 & 0.4256 \\
\hline $2^{*}$ & 38508250 & rs147711728 & $\mathrm{T}$ & 164 & 93 & 234 & $6.30 \mathrm{E}-06$ & 0.0108 \\
\hline 2 & 170990253 & rs185528286 & $\mathrm{C}$ & 159 & 91 & 226 & $4.45 \mathrm{E}-06$ & 0.0153 \\
\hline $3^{*}$ & 189410000 & rs2378515 & G & 54 & 34 & 73 & $5.29 \mathrm{E}-08$ & 0.1802 \\
\hline 4 & 170028965 & rs202156267 & A & -164 & -229 & -99 & $9.12 \mathrm{E}-07$ & 0.0313 \\
\hline 6 & 97632917 & rs148707864 & G & 140 & 79 & 200 & 7.01E-06 & 0.0148 \\
\hline $6^{*}$ & 130581980 & rs62431222 & A & 102 & 61 & 144 & $1.36 \mathrm{E}-06$ & 0.0329 \\
\hline 7 & 52698748 & rs150804725 & I & -112 & -159 & -64 & 4.65E-06 & 0.0297 \\
\hline 7 & 156412814 & rs849073 & $\mathrm{G}$ & -34 & -48 & -19 & $9.16 \mathrm{E}-06$ & 0.4351 \\
\hline $8^{*}$ & 1784364 & rs11988258 & A & -34 & -48 & -20 & $4.06 \mathrm{E}-06$ & 0.4201 \\
\hline 8 & 32613177 & rs75468268 & G & 155 & 87 & 223 & 8.95E-06 & 0.0134 \\
\hline 8 & 69224040 & rs814465 & $\mathrm{C}$ & 82 & 49 & 114 & $1.04 \mathrm{E}-06$ & 0.0537 \\
\hline 8 & 141147356 & rs142956251 & A & 155 & 87 & 224 & 8.43E-06 & 0.0139 \\
\hline $9^{*}$ & 21340235 & rs145072648 & $\mathrm{C}$ & 163 & 97 & 230 & $1.59 \mathrm{E}-06$ & 0.0126 \\
\hline 9 & 30859764 & rs138591003 & A & -178 & -255 & -100 & $7.76 \mathrm{E}-06$ & 0.0108 \\
\hline 10 & 97277536 & rs189712177 & G & 186 & 107 & 265 & 4.43E-06 & 0.0115 \\
\hline 10 & 128606691 & rs71490795 & A & 121 & 69 & 174 & $6.52 \mathrm{E}-06$ & 0.0225 \\
\hline 11 & 23509085 & rs148697259 & A & 133 & 75 & 192 & $9.22 \mathrm{E}-06$ & 0.0181 \\
\hline 11 & 99369450 & rs72991567 & G & 67 & 39 & 95 & $2.54 \mathrm{E}-06$ & 0.0804 \\
\hline 12 & 73510344 & rs60148685 & $\mathrm{D}$ & 34 & 19 & 48 & 8.09E-06 & 0.4437 \\
\hline 13 & 30571045 & rs200421910 & I & -64 & -92 & -36 & 8.33E-06 & 0.0988 \\
\hline 13 & 109383269 & rs151235076 & $\mathrm{T}$ & 188 & 108 & 267 & 4.27E-06 & 0.0097 \\
\hline 16 & 2390565 & rs45501400 & $\mathrm{T}$ & 169 & 94 & 243 & $9.89 \mathrm{E}-06$ & 0.0163 \\
\hline $17^{*}$ & 22020568 & rs201766885 & C & 113 & 65 & 161 & $4.24 \mathrm{E}-06$ & 0.0464 \\
\hline 19 & 53280252 & rs10414169 & $\mathrm{T}$ & 131 & 74 & 189 & 8.82E-06 & 0.0183 \\
\hline 22 & 32558640 & rs144964180 & A & 160 & 90 & 229 & $7.28 \mathrm{E}-06$ & 0.0122 \\
\hline 22 & 49801423 & rs9627723 & C & 45 & 26 & 64 & $4.15 \mathrm{E}-06$ & 0.3654 \\
\hline
\end{tabular}

* Tag-SNP in regions with $\geq 3$ SNPs with $p<10^{-5}$ for association with adipose morphology. Only results for SNPs with minor allele frequency (MAF) $>1 \%$ are shown. $\mathrm{I}=$ insertion; $\mathrm{D}=$ deletion. SNP, single nucleotide polymorphism, Chrom, chromosome, POS, position in the genome, ID, reference SNP accession number, BETA, beta coefficient, L95, lower $95 \%$ confidence interval, U95, upper $95 \%$ confidence interval, A1_FREQ, frequency of effect allele.

A)

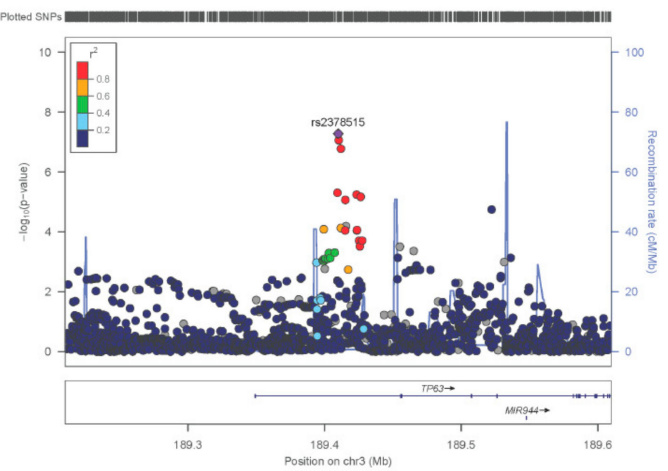

B)

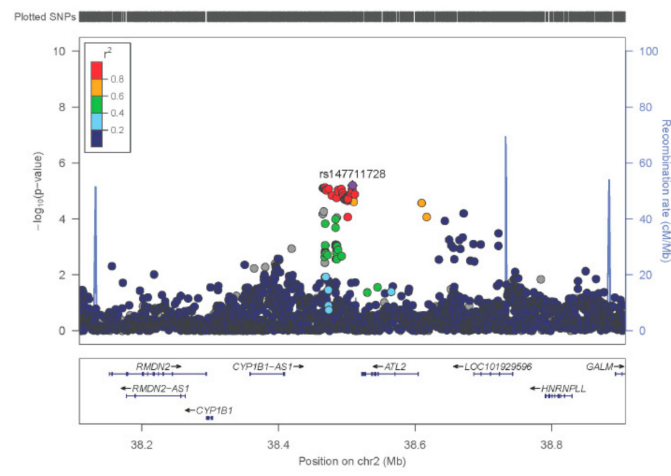

Figure 1. Cont. 
C)

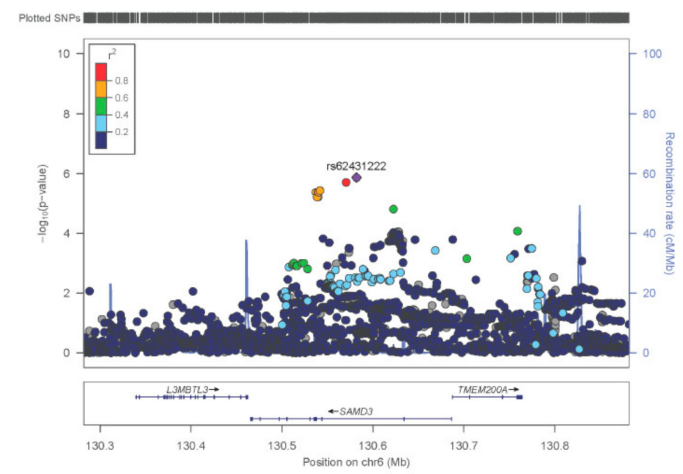

E)

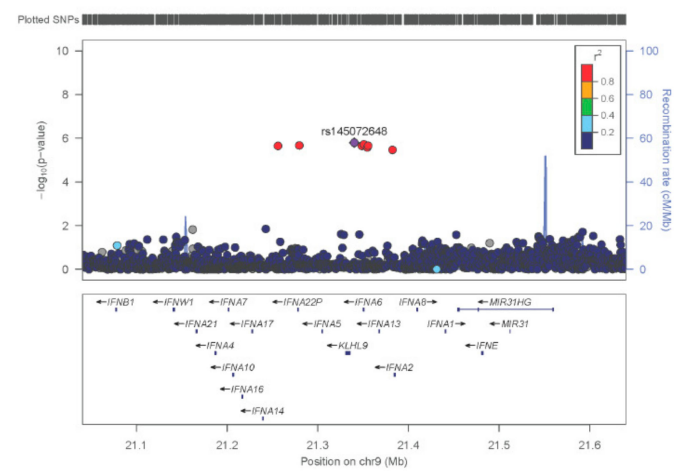

D)

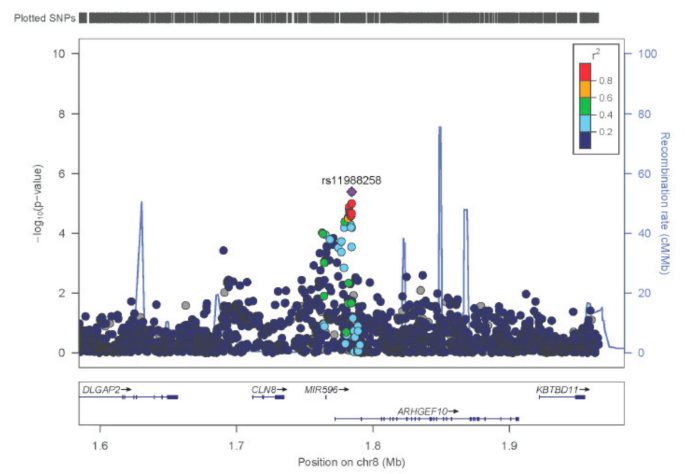

F)

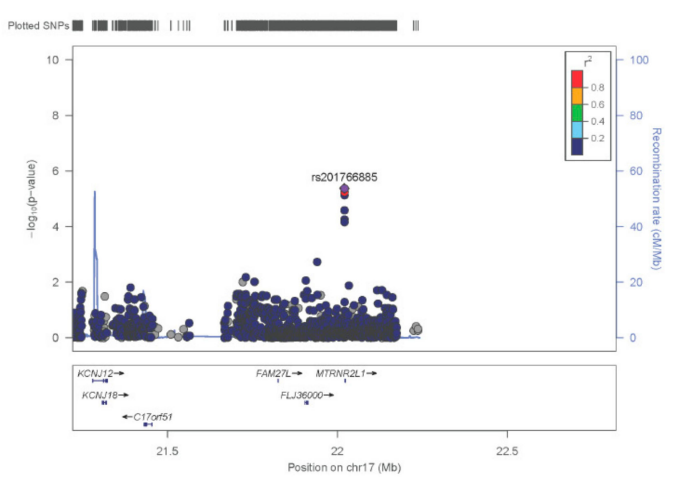

Figure 1. Genetic loci demonstrating suggestive association with adipose morphology.

\subsection{Functional Evaluation of Candidate Genes}

Transcriptome profiling of all protein-coding genes in the six adipose morphology-associated genetic loci revealed six genes from four loci, which were expressed in adipocytes and their progenitors isolated from SAT biopsies, as well as in hMSCs undergoing in vitro differentiation to adipocytes. (Figure 2A,B). The six genes were taken forward for functional evaluation in hMSCs to determine possible impact on adipogenesis. All genes except C17orf51 were enriched in progenitor cells as compared to mature adipocytes (Figure 2A). The expression of CYP1B1, TMEM200A, and ARHGEF10 decreased directly after induction of differentiation, whereas the expression of ATL2, C17orf51, and L3MBTL3 varied over the course of differentiation of hMSCs (Figure 2B).
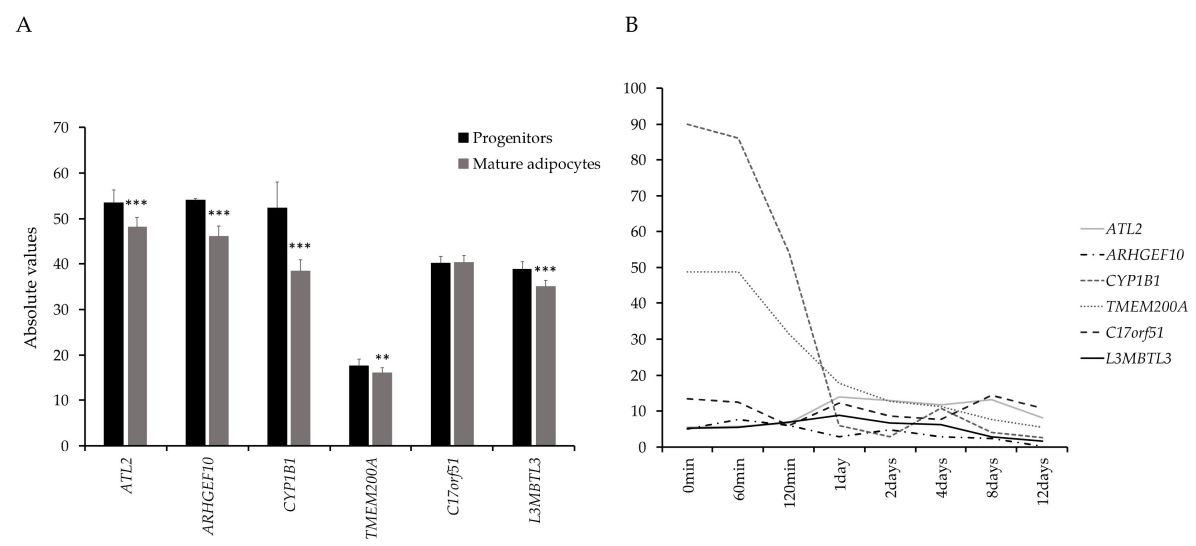

Figure 2. (A) Enrichment of candidate genes in progenitor cells and mature adipocytes. Data retrieved from the FANTOM5 dataset (http://fantom.gsc.riken.jp/5/) and Ehrlund, A et al. 2017 [29]. (B) Expression of candidate genes during human mesenchymal stem cell (hMSC) differentiation in vitro. 
Based on the expression pattern during differentiation, we knocked down the aforementioned candidate genes in hMSCs during proliferation, that is four days prior to induction of differentiation where day 0 corresponds to the start of differentiation. This resulted in a $60-80 \%$ decreased expression in all candidate genes except $L 3 M B T L 3$, where the efficiency of knockdown was 20-60\% (Figure 3A). We evaluated the accumulation of neutral lipids as a marker of adipocyte differentiation (Figure 3B, Figure S2) and the release of glycerol in cell culture medium as a marker of adipocyte lipolysis at day 9 of differentiation (Figure 3C). Both the accumulation of lipids and glycerol levels were reduced after knockdown of all six genes. We observed a $\sim 5-10 \%$ increase in cell number in knockdown of ATL2, ARHGEF10, CYP1B1, and TMEM200A and a modest reduction in cell number in knockdown of L3MBTL3 (Figure 4A). To investigate cell proliferation, we knocked down the candidate genes, and treated cells in each experiment with EdU, a marker of DNA synthesis in proliferating cells. The proportion of proliferating cells $\left(\mathrm{EdU}^{+}\right)$was $\sim 5-16 \%$ higher for all genes, although for L3MBTL3, the increase was modest (Figure 4B); however, this might be related to poor knockdown efficiency of L3MBTL3 (Figure 3A). Furthermore, the expression of the marker of proliferation Ki-67 ( $m$ Ki67) was increased day 0 before initiation of differentiation for all investigated genes (Figure 4C).
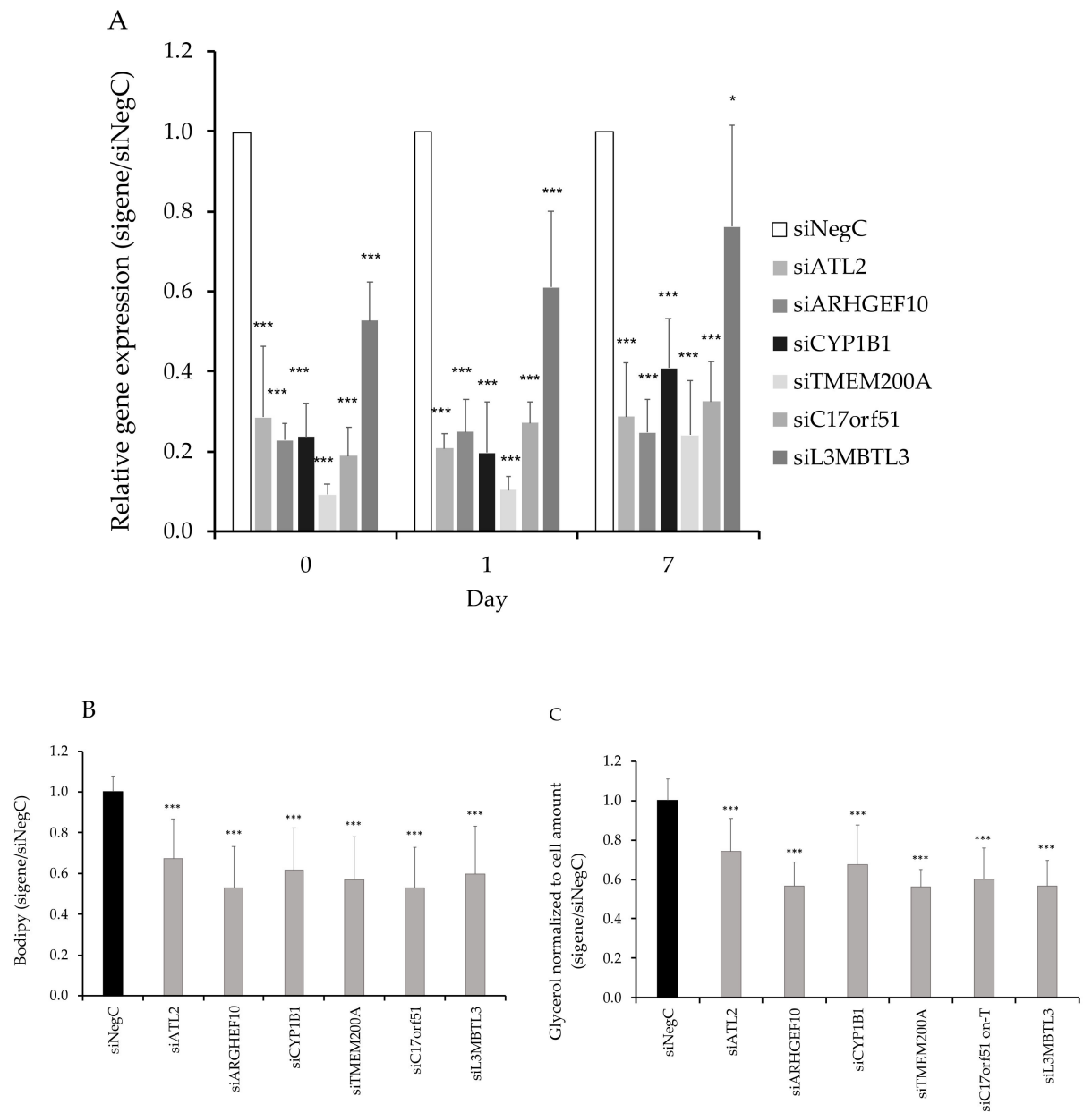

Figure 3. (A) ATL2, ARHGEF10, CYP1B1, TMEM200A, C17orf51, and L3MBTL3 was knocked down using small interfering RNAs (siRNA) in hMSCs four days (day -4) before induction of differentiation and followed until differentiation day 7 and 9. (B) Accumulation of neutral lipids was evaluated with Bodipy staining of cells at day 9. (C) Glycerol amount in the medium was measured, and all measured concentrations were within the range of the glycerol standard curve 0-120 $\mu \mathrm{M}$. Results were analyzed using a $t$-test and presented in fold change $\pm \mathrm{SD}$ relative to negative control of a corresponding time point (Neg C). ${ }^{* * *} p<0.005,{ }^{* *} p<0.01,{ }^{*} p<0.05$. 
A

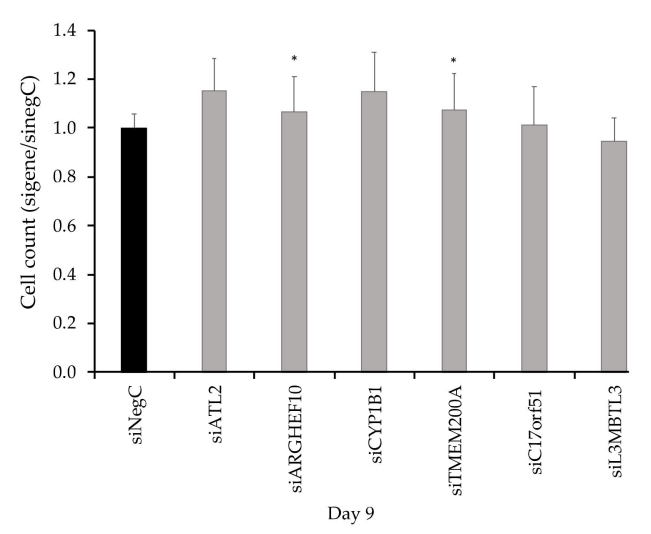

B

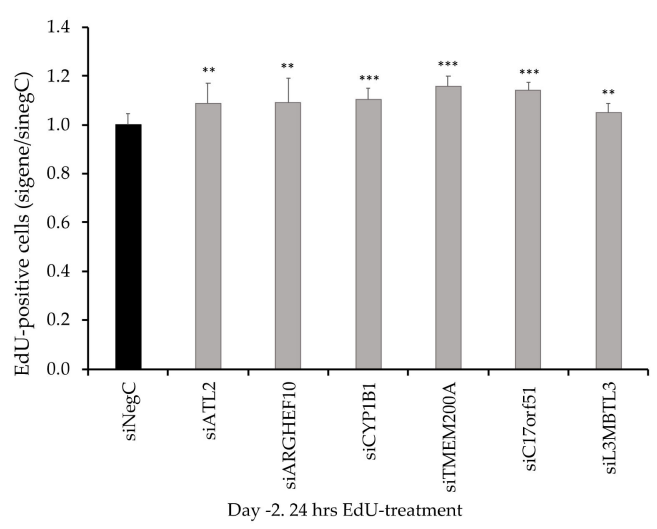

C

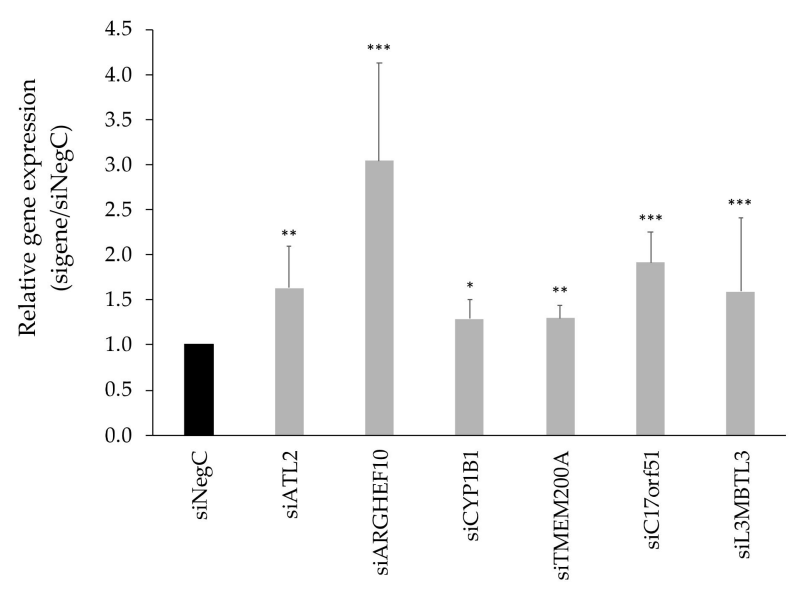

Figure 4. (A) Cell number at day 9 of differentiation was measured by Hoechst staining of nuclei. (B) Cell proliferation was evaluated by measuring DNA-synthesis with 5-ethynyl-2'-deoxyuridine (EdU). (C) mRNA-levels of proliferation marker $m$ KI67 were measured with real-time qPCR at day 0 before differentiation was initiated. Results were analyzed using a $t$-test and presented in fold change \pm SD relative to negative control of a corresponding time point (Neg C). ${ }^{* * *} p<0.005,{ }^{* *} p<0.01,{ }^{*} p<0.05$.

Expression levels of genes central to adipogenesis (peroxisome proliferator-activated receptor-gamma (PPARG), CCAAT/enhancer-binding protein alpha (CEBPA) and CCAAT/enhancer-binding protein beta $(C E B P B)$ and the adipocyte marker adiponectin $(A D I P O Q)$ were measured in each knockdown experiment (Figure 5) at differentiation start (day 0), day 1 day, and day 7 post differentiation induction. Knockdown of ATL2 resulted in a reduced expression of all adipogenesis genes (Figure 5A). ARHGEF10 knockdown resulted in a temporary modest reduction in the expression of $C E B P A$ and $C E B P B$ (Figure $5 \mathrm{~B})$. The knockdown of $C Y P 1 B 1$ resulted in reduced expression of PPARG, CEBPB, and $A D I P O Q$, and a modest reduction in CEBPA expression (Figure 5C). The knockdown of TMEM200A and L3MBTL3 resulted in a reduced expression of investigated adipogenesis genes, although the effect on PPARG expression was modest (Figure 5D,F). The knockdown of C17orf51 resulted in a strong reduction in ADIPOQ expression and a modest effect on the expression of adipogenesis genes (Figure 5E). 
A

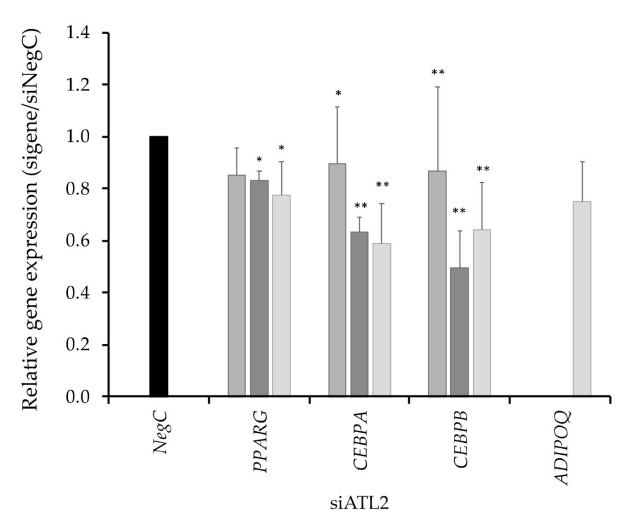

C

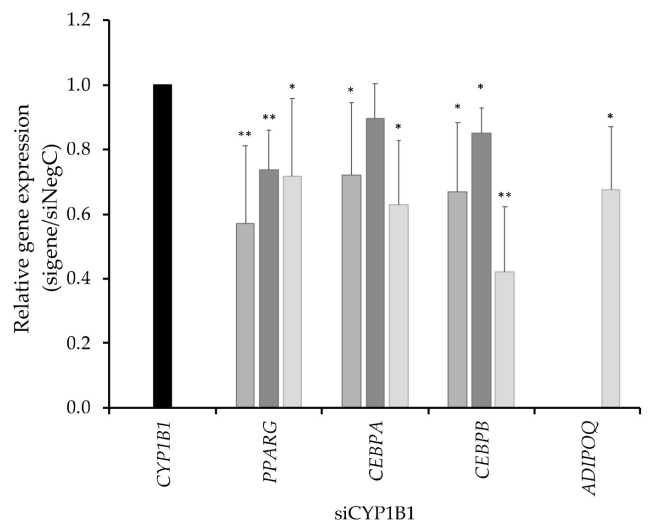

E

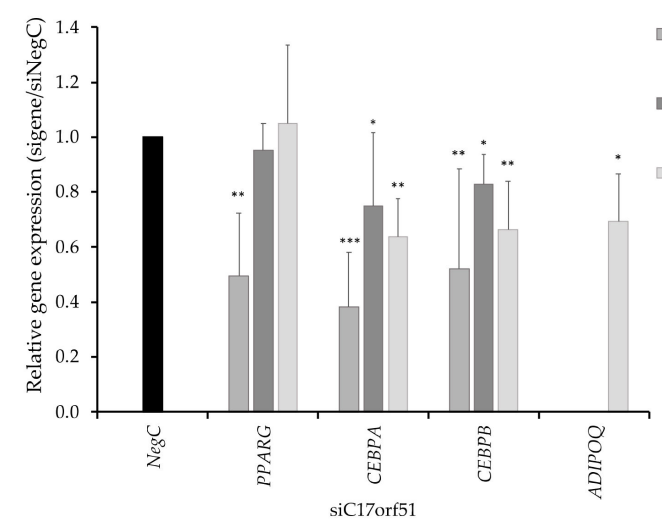

B
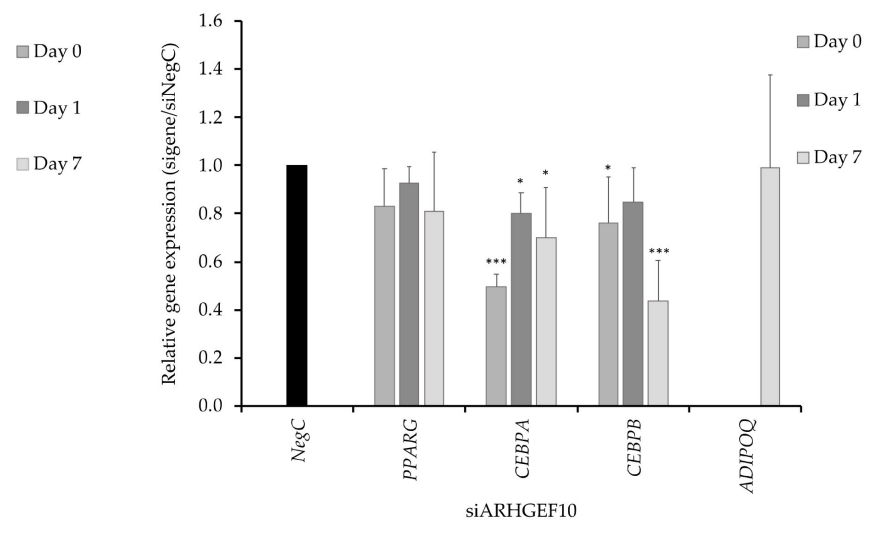

D

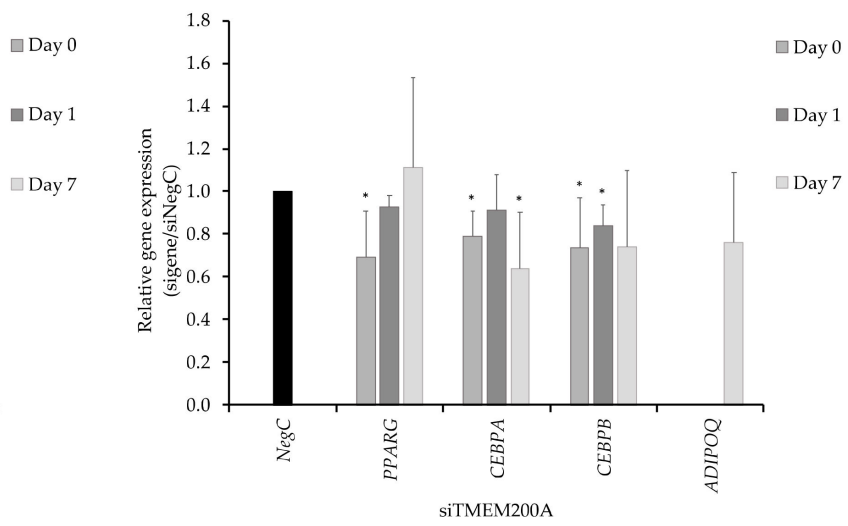

F

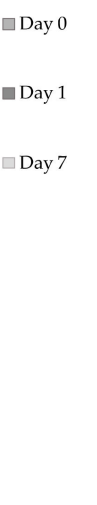

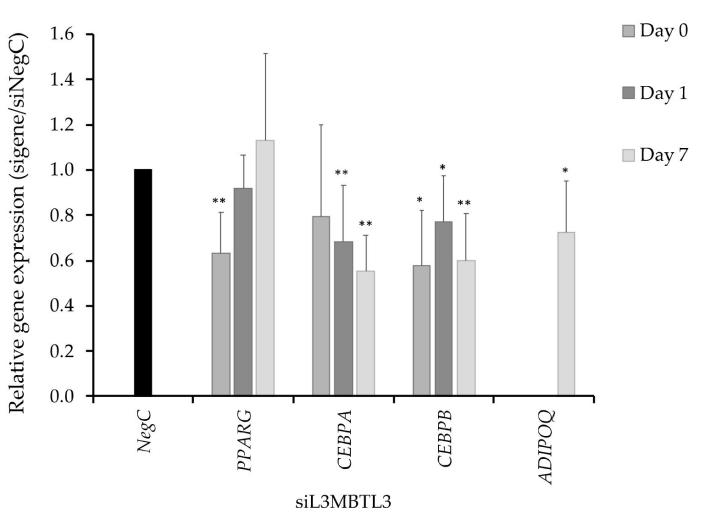

Figure 5. (A-F) The expression of peroxisome proliferator-activated receptor-gamma (PPARG), CCAAT/enhancer-binding protein alpha (CEBPA), and CCAAT/enhancer-binding protein beta (CEBPB) measured on day 0,1 , and 7 . The expression of $A D I P O Q$ was only measured at day 7 . Results are based on three biological/independent experiments. Expression of genes was normalized to the reference gene 18s. Results were analyzed using a $t$-test and presented in fold change \pm SD relative to negative control of a corresponding time point (Neg C). ${ }^{* *} p<0.005,{ }^{* *} p<0.01,{ }^{*} p<0.05$. 


\subsection{SAT-Expression of CYP1B1 and ATL2 Correlates With Adipose Morphology}

We next examined whether abdominal SAT expression of the six candidate genes was associated with adipose morphology using SAT-expression from a previously examined cohort of 114 women where adipose morphology was quantified as average adipocyte size in the abdominal SAT region adjusted for total body fat [16]. There was an inverse relationship between ATL2 expression and adipose morphology $\left(p 4.00 \times 10^{-4}, \mathrm{R}=0.33\right)$ and a positive association between CYP1B1 and adipose morphology $\left(p 3.01 \times 10^{-6}, \mathrm{R}=0.43\right)$. No association between ARHGEF10, TMEM200A, L3MBTL3, and C17orf51 and adipose morphology was observed.

\subsection{Overlap Between Result From GWAS Studies of T2D and Adipose Morphology}

None of the SNPs demonstrating suggestive association with adipose morphology have previously been associated with clinical metabolic traits, such as IR or T2D, according to the GWAS catalog (https://www.ebi.ac.uk/gwas/), February 14th, 2020). However, 25 genetic loci previously associated with T2D demonstrated nominal association with adipose morphology (Table 3). In 12 genetic loci, the allele increasing the risk of T2D was associated with adipose hypertrophy consistent with the finding that this adipose morphology increases the risk for T2D in genetic epidemiological studies [9]. None of these genetic loci encoded genes with established roles in human SAT. For the remaining loci, the risk allele is either not reported in the GWAS catalog, or the allele increasing the risk of T2D is associated with hyperplastic SAT making it difficult to interpret the finding. 
Table 3. Tag genome-wide association study (GWAS) SNPs associated with type 2 diabetes and with adipose morphology in the current study.

\begin{tabular}{|c|c|c|c|c|c|c|c|c|c|c|c|}
\hline \multirow[b]{2}{*}{ SNP } & \multirow[b]{2}{*}{ CHROM } & \multirow[b]{2}{*}{ POS } & \multicolumn{4}{|c|}{ GWAS Adipose Morphology } & \multicolumn{4}{|c|}{ GWAS Catalogue T2D } & \multirow{2}{*}{$\begin{array}{c}\text { Consistent Risk Alleles } \\
\text { For } \\
\text { Adipose Hypertrophy } \\
\text { and T2D }\end{array}$} \\
\hline & & & A1 & A1_FREQ & BETA & $P$ & PUBMED & Reported Genes & Risk Allele & OR BETA & \\
\hline rs12031188 & 1 & 51103268 & $\mathrm{C}$ & 0.40 & 15 & $4.50 \mathrm{E}-02$ & 30718926 & FAF1 & $\mathrm{C}$ & 1.08 & yes \\
\hline rs58432198 & 1 & 51256091 & $\mathrm{~T}$ & 0.11 & -24 & $4.58 \mathrm{E}-02$ & 30718926 & FAF1 & $\mathrm{C}$ & 1.10 & no \\
\hline rs2088315 & 3 & 162382517 & G & 0.50 & 16 & 3.27E-02 & 30130595 & OTOL1, LINC01192 & ? & 5.26 & not reported \\
\hline rs8192675 & 3 & 170724883 & $\mathrm{C}$ & 0.30 & 17 & 4.13E-02 & 28566273 & $N R$ & $\mathrm{~T}$ & 1.05 & no \\
\hline rs138306797 & 3 & 185545719 & $\mathrm{~T}$ & 0.02 & 76 & $1.32 \mathrm{E}-02$ & 25760438 & IGF2BP2 & ? & 0.78 & not reported \\
\hline rs9379084 & 6 & 7231843 & A & 0.10 & -48 & 2.94E-04 & 29632382 & RREB1 & G & 1.09 & yes \\
\hline rs9460550 & 6 & 20719561 & A & 0.16 & 26 & $1.01 \mathrm{E}-02$ & 31118516 & CDKAL1 & A & 1.15 & yes \\
\hline rs72892910 & 6 & 50816887 & $\mathrm{~T}$ & 0.20 & -18 & 4.61E-02 & 30054458 & TFAP2B & $\mathrm{T}$ & 0.06 & yes \\
\hline rs9384193 & 6 & 154554249 & $\mathrm{C}$ & 0.38 & -16 & 3.97E-02 & 28060188 & OPRM1, CNKSR3 & ? & & not reported \\
\hline rs10231619 & 7 & 43320594 & $\mathrm{~T}$ & 0.16 & 41 & 7.18E-05 & 25102180 & HECW1 & $\mathrm{T}$ & 1.13 & yes \\
\hline rs4729854 & 7 & 102383663 & A & 0.47 & -18 & $2.00 \mathrm{E}-02$ & 30595370 & & ? & & not reported \\
\hline rs896854 & 8 & 95960511 & $\mathrm{C}$ & 0.47 & 20 & 8.30E-03 & 20581827 & TP53INP1 & $\mathrm{T}$ & 1.06 & $\begin{array}{l}\text { no } \\
\text { no }\end{array}$ \\
\hline rs10761745 & 10 & 65101071 & G & 0.14 & -28 & $9.68 \mathrm{E}-03$ & 28406950 & $J M J D 1 C$ & $?$ & 1.20 & not reported \\
\hline rs4929965 & 11 & 2197286 & A & 0.37 & 16 & 4.17E-02 & 30718926 & INS, IGF2 & A & 1.12 & yes \\
\hline rs2237892 & 11 & 2839751 & $\mathrm{~T}$ & 0.07 & -47 & $1.12 \mathrm{E}-03$ & 22961080 & KCNQ1 & $\mathrm{C}$ & 1.32 & yes \\
\hline rs2722769 & 11 & 11228374 & G & 0.42 & -16 & $3.48 \mathrm{E}-02$ & 22238593 & $\begin{array}{l}\text { GALNTL4, } \\
\text { LOC729013 }\end{array}$ & $\mathrm{C}$ & 1.35 & unclear \\
\hline rs7931302 & 11 & 128236058 & $\mathrm{C}$ & 0.29 & 19 & $1.92 \mathrm{E}-02$ & 30054458 & ETS1 & $\mathrm{C}$ & 0.05 & yes \\
\hline rs11048456 & 12 & 26463082 & C & 0.26 & -17 & $4.66 \mathrm{E}-02$ & 30054458 & ITPR2 & C & 0.05 & yes \\
\hline rs1153188 & 12 & 55098996 & $\mathrm{~T}$ & 0.26 & 21 & 1.79E-02 & 18372903 & $D C D$ & A & 1.08 & no \\
\hline rs730570 & 14 & 101142890 & G & 0.18 & -20 & 3.74E-02 & 21573907 & C14orf70 & G & 1.14 & no \\
\hline rs1436955 & 15 & 62404382 & $\mathrm{~T}$ & 0.28 & -17 & 3.62E-02 & 20862305 & $C 2 C D 4 B$ & C & 1.13 & yes \\
\hline rs781852 & 17 & 3953102 & G & 0.36 & 17 & 3.43E-02 & 29632382 & ZZEF1 & G & 1.05 & yes \\
\hline rs11873305 & 18 & 58049192 & C & 0.04 & 54 & $1.04 \mathrm{E}-02$ & 22885922 & $M C 4 R$ & A & 1.18 & no \\
\hline rs7274168 & 20 & 32435978 & $\mathrm{C}$ & 0.41 & -20 & $8.28 \mathrm{E}-03$ & 30595370 & & $?$ & & not reported \\
\hline rs6017317 & 20 & 42946966 & G & 0.23 & 17 & 4.06E-02 & 22158537 & $\begin{array}{c}\text { FITM2, R3HDML, } \\
\text { HNF4A }\end{array}$ & G & 1.09 & yes \\
\hline rs16988991 & 20 & 42989777 & A & 0.20 & 17 & 4.63E-02 & 30718926 & $H N F 4 A$ & $\mathrm{~A}$ & 1.05 & yes \\
\hline rs2833610 & 21 & 33385186 & A & o & -17 & 2.75E-02 & 21490949 & HUNK & A & 1.17 & no \\
\hline
\end{tabular}

Where A1 = effect allele; GWAS, genome-wide association study, T2D, type 2 diabetes, SNP, single nucleotide polymorphism, CHROM, chromosome, POS, position in the genome, A1_FREQ, frequency of effect allele, BETA, beta coefficient, OR, Odds Ratio. 


\section{Discussion}

The present study has shed new light on the genetics of adipose morphology, identified six genetic loci harboring candidate genes, and provides evidence for these candidate genes as regulators of adipocyte renewal and lipid storage in adipose hypertrophy. Hypertrophic SAT morphology is a strong risk factor for IR and T2D. However, among established risk alleles for T2D from GWAS, only 12 alleles were nominally associated with hypertrophic adipose morphology.

In the adipose morphology-associated locus on chromosome 2, functional analysis by siRNA-mediated knockdown suggested CYP1B1 and/or ATL2 as potential causal links between genetic variants and adipose morphology. The knockdown of either gene stimulated proliferation of adipose-derived hMSCs accompanied by reduced expression of mRNA markers for adipogenesis, inhibited lipid accumulation, and reduced lipolysis in cells. Our interpretation of these findings is that a higher number of precursor cells resulted in a lower proportion of differentiating cells, i.e., reduced expression of adipogenesis markers and reduced lipid accumulation, rather than impaired adipogenesis. This interpretation is consistent with the finding that increased recruitment of new fat cells is a major determinant of adipose hyperplasia [4]. The expression pattern of CYP1B1 and ATL2 did not permit knockdown late in differentiation and, therefore, we could not determine whether these genes have a direct impact on mature fat cell lipid accumulation, or whether the lipid phenotype is secondary to a larger number of progenitor cells in the experiment. In either case, our findings support the hypothesis that CYP1B1 and ATL2 counteract a favorable hyperplastic adipose expansion by inhibiting the proliferation of precursor cells. The evidence for CYP1B1 was further supported by the finding of a positive association between SAT gene expression of CYP1B1 and hypertrophic adipose morphology in a large clinical cohort. CYP1B1 encodes a widely expressed monooxygenase involved in the metabolism of many important physiological compounds, including estrogen, arachidonic acid, melatonin, and retinoids [30]. CYP1B1-null mice display attenuated high-fat diet (HFD)-induced obesity and improved glucose tolerance related to increased fatty acid oxidation markers [31-33]. In mouse models, CYP1BI has been reported to control PPARG expression, whereas no impact on adipogenesis was observed [30]. The other candidate gene on chromosome 2, ATL2, has been reported to influence lipid droplets via the endoplasmatic reticulum [34]. The chromosome 2 locus, but not the SNPs reported herein, has previously been associated with BMI in GWAS [35]. Together previous and present findings give support to the notion that the chromosome 2 locus, possible via CYP1B1 and/or ATL2, controls the extent and fate of adipose expansion.

On chromosome 3, multiple SNPs in an intron of the TP63 gene displayed association with adipose morphology. Due to the high recombination in this locus, the association does not extend beyond the TP63 gene. TP63-- mice develop obesity and insulin resistance linked to increased fatty acid synthesis and decreased fatty acid oxidation [36]. None of the reported TP63 splice variants were detected at mRNA level in our in vitro system (adipose derived hMSCs) nor in mature adipocytes isolated from adipose tissue, and, therefore, we could not assess the function of TP63 in human adipocytes. Further characterization of the potential role of TP63 in human adipose tissue will need more refined methods that were beyond the scope of this study.

In the chromosome 6 locus, knockdown of L3MBTL3 and TMEM200A reduced lipid accumulation in fat cells. For TMEM200A, the finding was accompanied by the proliferation of adipose-derived hMSCs. The L3MBTL3 locus has recently been reported to be associated with systemic IR and lower levels of peripheral fat, and the encoded protein to stimulate adipocyte fat storage [10]. The herein reported association of the L3MBTL3 with hypertrophic adipose morphology potentially expands the knowledge of how this locus influences IR. We did not find any previous data on TMEM200A and metabolic disease; additional studies are needed to determine the importance of this gene for adipose function.

Finally, loci on chromosomes 8 and 17 were associated with adipose morphology. In these loci, knockdown of the ARHGEF10 and C17orf51 genes, respectively, promoted precursor cell proliferation and inhibited lipid accumulation, which could explain the association between these genetic loci and 
adipose morphology. ARHGEF10 belongs to the Rho guanine nucleotide exchange factor (GEF) family, which stimulates Rho GTPases and has been shown to have tumor suppressor activity and inhibits proliferation [37]. Beyond this, the function of these genes is poorly defined, and these genetic loci have not previously been implicated in the control of common metabolic disorders according to the GWAS catalog (https://www.ebi.ac.uk/gwas/).

SAT adipose morphology, as measured herein, categorizes the studied adipose depot as hypertrophic or hyperplastic, i.e., larger or smaller fat cells than expected given the size of the fat depot. In general, both average adipocyte volume and numbers increase with increasing BMI. An advantage with the applied measure of adipose morphology is that it takes the size of the fat depot into account. At the same time, adipose morphology, hereby, becomes a complex phenotypic outcome potentially dependent on genes controlling adipocyte number, lipid droplet size, as well as total body fat. In the present study, we chose to focus on precursor cell proliferation and lipid droplet size as potential functional links between candidate genes and adipose morphology. An appropriate functioning adipogenesis is essential for healthy lipid storage in subcutaneous AT. Inappropriate AT expansion, as observed with hypertrophic SAT, promotes ectopic lipid storage and systemic IR [10]. The reduced glycerol release observed after knockdown of candidate genes gives further support to the notion of impaired adipogenesis. The complexity of the phenotype might explain why most examined genes did not show an association between adipose gene expression and morphology.

One weakness of the present study is that we were unable to link SAT morphology associated SNPs functionally to specific genes, i.e., none of the morphology-associated SNPs were eQTLs in SAT or visceral adipose tissue or caused direct deleterious effects on gene function, e.g., missense mutations, according to bioinformatic analysis. Another weakness is the limited power of the study cohort. The latter is unavoidable for a phenotype, such as adipose volume, which is very resource-demanding to collect. However, the results of the functional exploration of genes in the identified morphology-associated loci provide validation for the approach. Finally, we only assessed SAT morphology although other adipose depots might be even more relevant to study [38], but are even less accessible for sampling.

In conclusion, our genetic and functional findings provide evidence for ATL2, ARHGEF10, CYP1B1, TMEM200A, C17orf51, and L3MBTL3 being important for adipose tissue morphology by regulation of proliferation and differentiation of precursor cells. Further work would be required to determine whether modulation of these genes could have therapeutic applications in IR and T2D, where adipogenesis is perturbed

Supplementary Materials: The following are available online at http://www.mdpi.com/2073-4409/9/5/1085/s1, Table S1. SNPs associated with adipose morphology with $p<10-5$.; Figure S1: QQ and Manhattan plots; Figure S2. Images of neutral lipids stained by Bodipy.

Author Contributions: R.J.S. designed the study, conducted analysis, interpreted the data, and edited the manuscript; V.L. conducted experiments, interpreted the data and drafted the manuscript; A.K. conducted experiments, interpreted the data, and edited the manuscript; I.D. and P.A. acquired the data, conceived and designed the study, interpreted the data, and edited the manuscript. All authors have agreed to be accountable for their contributions and have approved the final manuscript

Funding: R.J.S. is supported by a the UKRI innovation-HDR-UK Fellowship (MR/S003061/1). I.D. is supported by the Strategic Research Program in Diabetes at Karolinska Institutet (Genetic and long-term epigenetic studies of changes in adipose function), Swedish Research Council (2019-00997), Novo Nordisk foundation (The Role of Adipose Tissue in Cardiovascular Disease), and the Swedish Diabetes Association (DIA2019-407).

Data Availability: Summary GWAS is available upon request.

Conflicts of Interest: None.

\section{References}

1. Prins, J.B.; O'Rahilly, S. Regulation of adipose cell number in man. Clin. Sci. (Lond) 1997, 92, 3-11.

2. Hammarstedt, A.; Gogg, S.; Hedjazifar, S.; Nerstedt, A.; Smith, U. Impaired Adipogenesis and Dysfunctional Adipose Tissue in Human Hypertrophic Obesity. Physiol. Rev. 2018, 98, 1911-1941. [PubMed] 
3. Gustafson, B.; Hammarstedt, A.; Hedjazifar, S.; Smith, U. Restricted adipogenesis in hypertrophic obesity: The role of WISP2, WNT, and BMP4. Diabetes 2013, 62, 2997-3004. [PubMed]

4. Arner, E.; Westermark, P.O.; Spalding, K.L.; Britton, T.; Rydén, M.; Frisén, J.; Bernard, S.; Arner, P. Adipocyte turnover: Relevance to human adipose tissue morphology. Diabetes 2010, 59, 105-109. [PubMed]

5. Acosta, J.R.; Douagi, I.; Andersson, D.P.; Bäckdahl, J.; Rydén, M.; Arner, P.; Laurencikiene, J. Increased fat cell size: A major phenotype of subcutaneous white adipose tissue in non-obese individuals with type 2 diabetes. Diabetologia 2016, 59, 560-570. [PubMed]

6. Gao, H.; Mejhert, N.; Fretz, J.A.; Arner, E.; Lorente-Cebrián, S.; Ehrlund, A.; Dahlman-Wright, K.; Gong, X.; Strömblad, S.; Douagi, I. Early B cell factor 1 regulates adipocyte morphology and lipolysis in white adipose tissue. Cell Metab. 2014, 19, 981-992.

7. Minchin, J.E.; Dahlman, I.; Harvey, C.J.; Mejhert, N.; Singh, M.K.; Epstein, J.A.; Arner, P.; Torres-Vázquez, J.; Rawls, J.F. Plexin D1 determines body fat distribution by regulating the type V collagen microenvironment in visceral adipose tissue. Proc. Natl. Acad. Sci. USA 2015, 112, 4363-4368.

8. Andersson, D.P.; Dahlman, I.; Eriksson Hogling, D.; Bäckdahl, J.; Toft, E.; Qvisth, V.; Näslund, E.; Thorell, A.; Rydén, M.; Arner, P. Improved metabolism and body composition beyond normal levels following gastric bypass surgery: A longitudinal study. J. Intern. Med. 2019, 285, 92-101.

9. Arner, P.; Arner, E.; Hammarstedt, A.; Smith, U. Genetic predisposition for Type 2 diabetes, but not for overweight/obesity, is associated with a restricted adipogenesis. PLoS ONE 2011, 6, e18284.

10. Lotta, L.A.; Gulati, P.; Day, F.R.; Payne, F.; Ongen, H.; van de Bunt, M.; Gaulton, K.J.; Eicher, J.D.; Sharp, S.J.; Luan, J. Integrative genomic analysis implicates limited peripheral adipose storage capacity in the pathogenesis of human insulin resistance. Nat. Genet. 2017, 49, 17-26.

11. Andersson, D.P.; Arner, E.; Hogling, D.E.; Rydén, M.; Arner, P. Abdominal subcutaneous adipose tissue cellularity in men and women. Int. J. Obes. (Lond) 2017, 41, 1564-1569.

12. Kulyté, A.; Lundbäck, V.; Lindgren, C.M.; Luan, J.; Lotta, A.L.; Langenberg, C.; Arner, P.; Strawbridge, R.J.; Ingrid Dahlman, I. Genome-wide association study of adipocyte lipolysis in the GENetics of adipocyte lipolysis (GENiAL) cohort. Mol. Metab. 2020, 34, 85-96. [PubMed]

13. Gallagher, D.; Visser, M.; Sepúlveda, D.; Pierson, R.N.; Harris, T.; Heymsfield, S.B. How useful is body mass index for comparison of body fatness across age, sex, and ethnic groups? Am. J. Epidemiol. 1996, 143, 228-239. [PubMed]

14. Ryden, M.; Arner, P. Subcutaneous Adipocyte Lipolysis Contributes to Circulating Lipid Levels. Arterioscler. Thromb. Vasc. Biol. 2017, 37, 1782-1787.

15. Ryden, M.; Arner, P. Cardiovascular risk score is linked to subcutaneous adipocyte size and lipid metabolism. J. Intern. Med. 2017, 282, 220-228.

16. Dahlman, I.; Rydén, M.; Brodin, D.; Grallert, H.; Strawbridge, R.J.; Arner, P. Numerous Genes in Loci Associated With Body Fat Distribution Are Linked to Adipose Function. Diabetes 2016, 65, 433-437.

17. Kolaczynski, J.W.; Morales, L.M.; Moore, J.H., Jr.; Considine, R.V.; Pietrzkowski, Z.; Noto, P.F.; Colberg, J.; Caro, J.F. A new technique for biopsy of human abdominal fat under local anaesthesia with Lidocaine. Int. J. Obes. Relat. Metab. Disord. 1994, 18, 161-166.

18. Lofgren, P.; Andersson, I.; Wahrenberg, H.; Hoffstedt, J. No difference in lipolysis or glucose transport of subcutaneous fat cells between moderate-fat and low-fat hypocaloric diets in obese women. Horm. Metab. Res. 2005, 37, 734-740.

19. Hirsch, J.; Gallian, E. Methods for the determination of adipose cell size in man and animals. J. Lipid Res. 1968, 9, 110-119.

20. Hellmer, J.; Arner, P.; Lundin, A. Automatic luminometric kinetic assay of glycerol for lipolysis studies. Anal. Biochem. 1989, 177, 132-137.

21. Loh, P.R.; Danecek, P.; Palamara, P.F.; Fuchsberger, C.; A Reshef, Y.; K Finucane, H.; Schoenherr, S.; Forer, L.; McCarthy, S.; Abecasis, G.R.; et al. Reference-based phasing using the Haplotype Reference Consortium panel. Nat. Genet. 2016, 48, 1443-1448. [PubMed]

22. Chang, C.C.; Chow, C.C.; Tellier, L.C.; Vattikuti, S.; Purcell, S.M.; Lee, J.J. Second-generation PLINK: Rising to the challenge of larger and richer datasets. Gigascience 2015, 4, 7. [PubMed]

23. Watanabe, K.; Taskesen, E.; van Bochoven, A.; Posthuma, D. Functional mapping and annotation of genetic associations with FUMA. Nat. Commun. 2017, 8, 1826. [PubMed]

24. GTEx Consortium. The Genotype-Tissue Expression (GTEx) project. Nat. Genet. 2013, 45, 580-585. 
25. Pettersson, A.M.; Stenson, B.M.; Lorente-Cebrián, S.; Andersson, D.P.; Mejhert, N.; Krätzel, J.; Aström, G.; Dahlman, I.; Chibalin, A.V.; Arner, P.; et al. LXR is a negative regulator of glucose uptake in human adipocytes. Diabetologia 2013, 56, 2044-2054.

26. Arner, E.; Daub, C.O.; Vitting-Seerup, K.; Andersson, R.; Lilje, B.; Drabløs, F.; Lennartsson, A.; Rönnerblad, M.; Hrydziuszko, O.; Vitezic, M.; et al. Transcribed enhancers lead waves of coordinated transcription in transitioning mammalian cells. Science 2015, 347, 1010-1014.

27. Lundback, V.; Kulyte, A.; Strawbridge, R.J.; Ryden, M.; Arner, P.; Marcus, C.; Dahlman, I. FAM13A and POM121C are candidate genes for fasting insulin: Functional follow-up analysis of a genome-wide association study. Diabetologia 2018, 61, 1112-1123.

28. Livak, K.J.; Schmittgen, T.D. Analysis of relative gene expression data using real-time quantitative PCR and the 2(-Delta Delta C(T)) Method. Methods 2001, 25, 402-408.

29. Ehrlund, A.; Acosta, J.R.; Björk, C.; Hedén, P.; Douagi, I.; Arner, P.; Laurencikiene, J. The cell-type specific transcriptome in human adipose tissue and influence of obesity on adipocyte progenitors. Sci. Data 2017, 4, 170164.

30. Li, F.; Zhu, W.; Gonzalez, F.J. Potential role of CYP1B1 in the development and treatment of metabolic diseases. Pharmacol. Ther. 2017, 178, 18-30.

31. Liu, X.; Huang, T.; Li, L.; Tang, Y.; Tian, Y.; Wang, S.; Fan, C. CYP1B1 deficiency ameliorates obesity and glucose intolerance induced by high fat diet in adult C57BL/6J mice. Am. J. Transl. Res. 2015, 7, 761-771. [PubMed]

32. Larsen, M.C.; Bushkofsky, J.R.; Gorman, T.; Adhami, V.; Mukhtar, H.; Wang, S.; Reeder, S.B.; Sheibani, N.; Jefcoate, C.R. Cytochrome P450 1B1: An. unexpected modulator of liver fatty acid homeostasis. Arch. Biochem. Biophys. 2015, 571, 21-39. [PubMed]

33. Li, F.; Jiang, C.; Larsen, M.C.; Bushkofsky, J.; Krausz, K.W.; Wang, T.; Jefcoate, C.R.; Gonzalez, F.J. Lipidomics reveals a link between CYP1B1 and SCD1 in promoting obesity. J. Proteome Res. 2014, 13, 2679-2687. [PubMed]

34. Le Guillou, S.; Laubier, J.; Péchoux, C.; Aujean, E.; Castille, J.; Leroux, C.; Le Provost, F. Defects of the endoplasmic reticulum and changes to lipid droplet size in mammary epithelial cells due to miR-30b-5p overexpression are correlated to a reduction in Atlastin 2 expression. Biochem. Biophys. Res. Commun. 2019, 512, 283-288.

35. Akiyama, M.; Okada, Y.; Kanai, M.; Takahashi, A.; Momozawa, Y.; Ikeda, M.; Iwata, N.; Ikegawa, S.; Hirata, M.; Matsuda, K.; et al. Genome-wide association study identifies 112 new loci for body mass index in the Japanese population. Nat. Genet. 2017, 49, 1458-1467. [PubMed]

36. Su, X.; Gi, Y.J.; Chakravarti, D.; Chan, I.L.; Zhang, A.; Xia, X.; Tsai, K.Y.; Flores, E.R. TAp63 is a master transcriptional regulator of lipid and glucose metabolism. Cell. Metab. 2012, 16, 511-525. [PubMed]

37. Joseph, J.; Radulovich, N.; Wang, T.; Raghavan, V.; Zhu, C.Q.; Tsao, M.S. Rho guanine nucleotide exchange factor ARHGEF10 is a putative tumor suppressor in pancreatic ductal adenocarcinoma. Oncogene 2020, 39, 308-321.

38. Hoffstedt, J.; Arner, E.; Wahrenberg, H.; Andersson, D.P.; Qvisth, V.; Löfgren, P.; Rydén, M.; Thörne, A.; Wirén, M.; Palmér, M.; et al. Regional impact of adipose tissue morphology on the metabolic profile in morbid obesity. Diabetologia 2010, 53, 2496-2503.

(C) 2020 by the authors. Licensee MDPI, Basel, Switzerland. This article is an open access article distributed under the terms and conditions of the Creative Commons Attribution (CC BY) license (http://creativecommons.org/licenses/by/4.0/). 